\title{
O Processo de Análise Hierárquica (AHP) e a teoria das Inteligências Múltiplas (MI): uma revisão de literatura com meta-síntese sobre a relação entre o método e a teoria
}

\author{
Carlos Eduardo Pereira de Quadros ${ }^{1}$ \\ Programa de Pós-Graduação em Modelagem Computacional - PPGMC, Rio Grande, RS \\ Diana Francisca Adamatti ${ }^{2}$ \\ PPGMC, Rio Grande, RS \\ André Andrade Longaray ${ }^{3}$ \\ PPGMC, Rio Grande, RS
}

Resumo. Este trabalho traz uma investigação, através de uma revisão sistemática e meta-síntese, acerca de artigos acadêmicos que trabalharam com o Processo de Análise Hierárquica (AHP) em conjunto com a teoria das Inteligências Múltiplas (MI). A busca através das palavras-chave "Analytic Hierarchy Process" e "Multiple Intelligences" retornou 107 trabalhos e, desses, apenas sete realmente trabalhavam com o método AHP e a teoria das MI. A apresentação desses trabalhos foi feita através de uma meta-síntese para elucidar sobre quais áreas foram empregados, suas conclusões e resultados.

Palavras-chave. Processo de Análise Hierárquica, Inteligências Múltiplas, Revisão de trabalhos, Meta-síntese

\section{Introdução}

Essa pesquisa faz uma investigação de artigos que utilizaram o Processo de Análise Hierárquica - AHP (Analytic Hierarchy Process) e a teoria das Inteligências Múltiplas - MI (Multiple Intelligences) de forma conjunta.

O Processo de Análise Hierárquica (AHP) foi criado pelo professor Thomas L. Saaty em 1980 [13]. O AHP é um método de tomada de decisão multicritério, baseado na hierarquia do grau de importância de um critério sobre os demais, que tem sido usado em muitas aplicações relacionadas com a tomada de decisão. A ideia principal deste método é dividir o problema de decisão em níveis hierárquicos para facilitar a compreensão e avaliação. Durante o processo de avaliação, este método permite o uso de critérios qualitativos e quantitativos. Saaty [14] descreve de forma didática como devemos estruturar a decisão de um problema, como montar as escalas que farão parte da hierarquia entre fatores mais e menos importantes, entre outros pontos importantes do AHP. O trabalho de Vaidya [17] traz uma visão geral das aplicações que usam o método multicritério e os trabalhos são categorizados de acordo com os temas identificados com base nas áreas de aplicação, agrupados por ano e também por região. De modo mais refinado, Saaty [15] apresenta um trabalho que mostra que os julgamentos de determinados atributos podem ser inconsistentes e, para sanar esses problemas de comparações que são feitas por meio de uma escala de julgamentos absolutos,

\footnotetext{
${ }^{1}$ carlos.quadros@furg.com

2 dianaada@gmail.com

3 andrelongaray@furg.br
} 
ele explora no trabalho algumas preocupações do método, como melhorar os julgamentos e quando possível obter melhor consistência nos julgamentos.

A teoria das Inteligências Múltiplas foi criada em 1983 por Howard Gardner através de seu livro Estruturas da mente: a teoria das inteligências múltiplas [3]. Nesse livro o autor detalha cada uma das inteligências da teoria e, no início, eram sete inteligências e posteriormente a oitava inteligência foi inserida. Nesse contexto, a teoria das MI conta atualmente com as seguintes inteligências: Musical, Corporal-cinestésica, Lógico-matemática, Linguística, Espacial, Interpessoal, Intrapessoal e Naturalista. Cada uma das inteligências engloba certas características e essas características se prestam a profissões particulares, o trabalho também sugere maneiras específicas para os educadores incorporarem as inteligências em seus planejamentos de aulas diárias para uso prático em sala de aula [8]. Visser [18] propôs um trabalho que executava dois testes em cada uma das oito inteligências e, nessa investigação, a análise fatorial revelou um grande fator "g" com cargas substanciais para testes que avaliam habilidades puramente cognitivas - linguística, lógico-matemática, espacial, naturalística, interpessoal - mas cargas mais baixas para testes de outras habilidades, especialmente corporal-cinestésica. Armstrong [2] usa o termo "personalização" para se referir a projetos e atividades centrados no aluno e dirigidos ao aluno, que enfatizam fortemente a voz e a escolha do aluno. Nessa proposta, os alunos precisam avaliar francamente seus próprios pontos fortes e fracos, se engajar no estabelecimento de metas realistas e ajustar suas metas conforme o projeto se desenvolve. Da mesma forma, em projetos de equipe personalizados, os alunos devem aprender como colaborar e participar da troca necessária para implementar de maneira eficaz seus planos e visualizar as conexões sociais necessárias para atingir seus objetivos. No livro Inteligências múltiplas ao redor do mundo, Gardner, Chen e Moran [5] apresentam como ocorre a aplicação da teoria das MI em diversos continentes, culturas e ambientes diversificados através da descrição, análise, avaliação e síntese. Portanto, a ideia principal desta revisão de literatura e meta-síntese é descobrir como se relacionam o método AHP e a teoria das MI através da revisão dos trabalhos acadêmicos encontrados.

\section{Metodologia}

Utilizando a base de dados acadêmicos Google Scholar no período de dezembro de 2020 e adotando os seguintes critérios de busca: período, a qualquer momento; classificação, por relevância; idioma, todos; termos de busca, "analytic hierarchy process" e "multiple intelligences", essa pesquisa teve o retorno de cento e dezessete trabalhos acadêmicos. Mesmo acessando através do Portal de Periódicos Capes, que tem conteúdo assinado e disponível para os acessos com endereço de protocolo da internet (IP) identificado para as instituições participantes, não houve possibilidade de resgatar todos os cento e dezessete trabalhos que a busca retornou.

O número de trabalhos acessíveis que incluía o arquivo em formato PDF (Portable Document Format) para ser usado na pesquisa foi de sessenta e cinco. Foram excluídos da coleta dissertações e teses, ficando apenas com artigos científicos. Foram aceitos para essa revisão apenas trabalhos escritos na língua inglesa. E, por fim, após ser feita uma leitura nesses trabalhos, apenas sete utilizavam o Processo de Análise Hierárquica (AHP) em conjunto com a Teoria das Inteligências Múltiplas (IM).

A partir desses sete trabalhos essa pesquisa fez uma descrição sucinta de cada um e criou um estudo de meta-síntese para expor seus resultados.

\section{Resultados obtidos}

Em um primeiro momento, os trabalhos resgatados nesta pesquisa foram colocados em uma taxonomia de acordo com a ordem cronológica de sua publicação. A síntese de dados envolve a comparação e o resumo dos resultados dos estudos primários incluídos [6]. Após isso, a pesquisa 
apresenta alguns resultados e discussões acerca dos trabalhos envolvidos encontrados através dos critérios aplicados descritos na metodologia.

O trabalho desenvolvido por Rezaie et al. [10], propôs uma estrutura conceitual para classificar as múltiplas inteligências de pessoas com epilepsia. O modelo inverso do Teste de Habilidade Em Epilepsia (ATIE), Fuzzy Inverse ATIE (FIA) foi desenvolvido para obter os melhores parâmetros de inteligência que seriam obtidos para maximizar a probabilidade de emprego de pessoas com epilepsia (PWE). A estrutura proposta considerou tanto fatores quantitativos quanto qualitativos. O método qualitativo foi usado para comparar os critérios qualitativos das alternativas, enquanto o modelo de análise multicritério (MCDEA) pode ser usado para melhorar o poder discriminante do método Análise Envoltória de Dados (DEA) clássico.

Em 2013, na Malásia, Rezaie et al. [11] apresentaram o trabalho "Classificando as Múltiplas Inteligências de Pessoas com Epilepsia Usando o Processo de Análise Hierárquica e Análise de Envolvimento de Dados" que trazia um método integrado de Processo de Análise Hierárquica e Envoltória de Dados (AHP-DEA) para classificar os parâmetros de inteligência de pessoas com epilepsia (PWE). O método proposto serviu para classificar as inteligências sugeridas que precisavam ser melhoradas, obtidas no Teste de Habilidade Em Epilepsia (ATIE), um teste psicométrico baseado na Inteligência Múltipla de Howard Gardner (MI). O processo foi feito com base no AHP com quatro etapas: modelagem do problema, avaliação de pesos, agregação de pesos e análise de sensibilidade. Neste trabalho, uma estrutura conceitual foi proposta para construir um método AHP-DEA integrado para classificar os parâmetros de inteligência de PWE que incluíam fatores qualitativos e quantitativos. Para investigar os efeitos dos aspectos demográficos nos parâmetros de inteligência, o AHP foi útil para a análise de sensibilidade, que pode ser realizada em três aspectos: pesos, prioridades locais e comparações.

Rezaie et al. [12] apresentaram um trabalho que tinha o objetivo de avaliar e classificar as inteligências múltiplas para pessoas com epilepsia (PWE). A metodologia do trabalho é dividida em dois passos principais. Primeiro, foi preciso identificar os efeitos dos aspectos demográficos, como nível educacional, status de empregabilidade, gênero, tipo de convulsão, etnia e situação conjugal nos parâmetros de inteligência. O processo foi feito com base no AHP com as seguintes etapas: modelagem do problema, avaliação de pesos, agregação de pesos e análise de sensibilidade. Segundo, foi necessário aplicar o modelo integrado AHP-DEA para derivar o método de classificação AHP-DEA mais favorável para os parâmetros de inteligência. Nessa etapa, cada paciente foi considerado uma Unidade de tomada de decisão (DMU). Portanto, a idade de cada paciente foi considerada como entrada para cada DMU. Dessa forma, a idade de início e os pesos obtidos na etapa um para cada parâmetro de inteligência considerando os efeitos dos aspectos demográficos foram considerados como saída para cada DMU. A saída desta etapa retornava a classificação priorizada para os parâmetros de inteligência considerando os efeitos dos aspectos demográficos.

Em Taiwan, Liao et al. [7] apresentaram um trabalho que visava construir indicadores de competência, integrando a aprendizagem baseada em investigação no estudo de projeto dos alunos em escolas de ensino médio profissionalizantes. Foi desenvolvida uma escala com base nas ideias e conceitos derivados da revisão da literatura, e a distribuição do peso dos itens da escala foi determinada por meio do AHP. Como conclusão, o estudo apontou uma série de dificuldades na aprendizagem baseada em investigação, e também sugeriu algumas recomendações para o Ministério da Educação, para professores e para pesquisas ou práticas futuras. Como exemplo de sugestão, a integração da aprendizagem baseada na investigação ao estudo do projeto dos alunos, a promoção das habilidades dos alunos em pensamento criativo, resolução de problemas, adoção de mudanças sociais e autodesenvolvimento, em resposta ao espírito de investigação científica.

Em 2018, na Indonésia, Oktavia e Madyatmadja [9] utilizaram o Processo de Análise Hierárquica (AHP) e a Técnica de Preferência de Pedido por Similaridade à Solução Ideal (TOPSIS) para propor um modelo capaz de sugerir uma recomendação na seleção de uma concentração com base nas 
habilidades dos alunos e facilitar a instituição de ensino superior na determinação da concentração (interesses preferidos) dos mesmos. Nesse modelo, os critérios para escolher a concentração foram inteligências múltiplas, média das notas, pontuação do curso e pontuação do TOEFL (Test of English as a Foreign Language). Como resultado na base de testes de precisão que foram feitos, o modelo obteve uma taxa de precisão de $67 \%$. Portanto, este sistema de suporte à decisão precisa ser avaliado mais detalhadamente porque a validade e confiabilidade não puderam ser garantidas e requerem mais amostras. E por fim, depois de utilizar o modelo de aplicação, os alunos puderam consultar o departamento da Universidade para que a concentração escolhida realmente se adaptasse e pudesse apoiar os alunos no futuro.

Ahsan et al. [1] apresentaram um trabalho com o objetivo de decidir e classificar a inteligência do aluno com base na teoria das Inteligências Múltiplas. O trabalho é baseado em quatro métodos, que são: Produto Ponderado (WP), Processo de Análise Hierárquica (AHP), Método de Ponderação Aditiva Simples (SAW), Eliminação e Escolha como expressão da realidade (ELimination Et Choix REAL TRADICIONAL - ELECTRE), Técnica de Preferência de Pedido por Similaridade à Solução Ideal (TOPSIS). Na primeira etapa o método AHP adotou uma estrutura hierárquica que começou com o objetivo principal e na segunda etapa avaliou cada critério. Posteriormente, fez uma matriz de comparação e na próxima avaliação calculou os autovetores de cada matriz para retornar a consistência hierárquica. Segundo os autores, os resultados do método de decisão podem representar as decisões de um especialista na determinação da inteligência infantil com base na Inteligência Múltipla e podem ajudar um professor orientador a ver como os alunos aprendem.

O trabalho elaborado por Yan [19] propôs um modelo chamado de "segunda sala de aula" para estimular a capacidade inovadora de estudantes universitários de Matemática. Em conjunto com este modelo foi construído um sistema de avaliação da capacidade de inovação dos estudantes universitários com base na atividade da segunda sala de aula de matemática. Este sistema, por sua vez, tem como base o Processo de Análise Hierárquica. A capacidade de inovação dos estudantes universitários, que foi avaliada neste trabalho, é dividida em quatro partes: a capacidade de adquirir conhecimento, a capacidade de pensamento ágil, a capacidade de aplicação prática e a capacidade de comunicação social. A segunda sala de aula (modelo apresentado no trabalho) e a sala de aula convencional trabalharam de forma harmoniosa aprimorando o crescimento profissional de toda a equipe envolvida. Em conclusão, o pesquisador descreveu que quanto mais os alunos participavam das atividades da segunda sala de aula de matemática, melhores eram os resultados da avaliação das suas capacidades inovadoras.

O objetivo da meta-síntese qualitativa é levar em conta todas as semelhanças e diferenças importantes na linguagem, conceitos, imagens e outras ideias em torno de uma experiência alvo [16]. Dessa forma, o Quadro 1 apresenta os principais pontos para análise.

Dos sete trabalhos encontrados, três são do mesmo grupo de pesquisadores de Rezaie et al. Este grupo de pesquisadores utiliza AHP e a teoria das MI para investigar potencialidades da teoria através do método envolvendo pacientes com epilepsia.

A relação de frequência dos trabalhos encontrados na investigação é baixa. De 2012 em diante temos apenas um trabalho encontrado por ano, com ausências nos anos de 2015 e 2017, onde não foram encontrados nenhum trabalho. Apenas no ano de 2019 é que encontramos dois trabalhos.

Os trabalhos recuperados através da pesquisa apresentaram atuação em apenas duas áreas de concentração. Dos sete trabalhos, três foram aplicados na área da saúde (todos do grupo Rezaie et al) e quatro possuem aplicação na área da educação. Cabe observar que a teoria das MI tem suas raízes na área da educação, mas isso não impede que ela seja aplicada em outras áreas de concentração. E, também, dos quatro trabalhos apresentados para a área de educação, dois tiveram resultados positivos para suas propostas e dois tiveram resultados não tão satisfatórios em relação às suas propostas.

Além do método de AHP empregado nas pesquisas encontradas, relacionado à teoria das IM, 
Quadro 1: Síntese dos dados de trabalhos encontrados na pesquisa

\begin{tabular}{|c|c|c|c|c|}
\hline Autores - Ano & Objetivo & Metodologia & Resultado & Conclusão \\
\hline $\begin{array}{l}\text { Rezaie V, Ah- } \\
\text { mad T, Maan } \\
\text { N, Awang SR, } \\
\text { Khanmoham- } \\
\text { madi M - } \\
2012 \text { - }\end{array}$ & $\begin{array}{l}\text { Classificar as inte- } \\
\text { ligências } \\
\text { obtidas noridas } \\
\text { (Fuzzy no FIA } \\
\text { ATIE) }\end{array}$ & $\begin{array}{l}\text { AHP, MCDM } \\
\text { (Multiple } \\
\text { Criteria Deci- } \\
\text { sion Making), } \\
\text { DEA (Data } \\
\text { Envelopment } \\
\text { Analysis), } \\
\text { ATIE (Abi- } \\
\text { lity Test in } \\
\text { Epilepsy), FIA }\end{array}$ & $\begin{array}{l}\text { Criação de uma es- } \\
\text { trutura conceitual para } \\
\text { resolver um problema } \\
\text { complexo de tomada } \\
\text { de decisão multicritério } \\
\text { com fatores qualitati- } \\
\text { vos. }\end{array}$ & $\begin{array}{l}\text { O método qualitativo } \\
\text { foi usado para comparar } \\
\text { critérios qualitativos das } \\
\text { alternativas, enquanto o } \\
\text { modelo MCDEA pode } \\
\text { ser usado para melhorar } \\
\text { o poder discriminante do } \\
\text { método DEA clássico }\end{array}$ \\
\hline $\begin{array}{l}\text { Rezaie V, Ah- } \\
\text { mad T, Maan } \\
\text { N, Awang SR, } \\
\text { Khanmoham- } \\
\text { madi M - } \\
2013\end{array}$ & 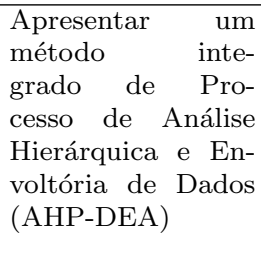 & $\begin{array}{l}\text { AHP, DEA, } \\
\text { ATIE, FIA }\end{array}$ & $\begin{array}{l}\text { Construção de um } \\
\text { método AHP-DEA in- } \\
\text { tegrado que classificou } \\
\text { parâmetros de inte- } \\
\text { ligência de pessoas com } \\
\text { epilepsia que incluem } \\
\text { fatores qualitativos e } \\
\text { quantitativos }\end{array}$ & $\begin{array}{l}\text { A análise de sensibilidade } \\
\text { pode mostrar a robustez } \\
\text { da decisão tomada por } \\
\text { meio do método }\end{array}$ \\
\hline $\begin{array}{l}\text { Rezaie V, Ah- } \\
\text { mad T, Awang } \\
\text { SR, Khan- } \\
\text { mohammadi } \\
\text { M, Maan N - } \\
\text { 2014 }\end{array}$ & $\begin{array}{l}\text { Avaliar e classifi- } \\
\text { car as inteligências } \\
\text { múltiplas para pes- } \\
\text { soas com epilepsia } \\
\text { (PWE) }\end{array}$ & $\begin{array}{l}\text { AHP, DEA, } \\
\text { ATIE, FIA }\end{array}$ & $\begin{array}{l}\text { O estudo determi- } \\
\text { nou a prioridade de } \\
\text { oito parâmetros de } \\
\text { habilidades de inte- } \\
\text { ligência, considerando } \\
\text { a demografia da pes- } \\
\text { soa com epilepsia e } \\
\text { usou essa prioridade } \\
\text { para aumentar a em- } \\
\text { pregabilidade dessas } \\
\text { pessoas. }\end{array}$ & $\begin{array}{l}\text { Com o conhecimento } \\
\text { da avaliação de desem- } \\
\text { penho e classificação } \\
\text { dos parâmetros de in- } \\
\text { teligência para pessoas } \\
\text { com epilepsia, a pesquisa } \\
\text { conseguiu identificar os } \\
\text { pontos fortes e fracos no } \\
\text { contexto de empregabi- } \\
\text { lidade no mercado de } \\
\text { trabalho e melhorar suas } \\
\text { competências inerentes } \\
\text { para buscar um emprego } \\
\text { mais adequado }\end{array}$ \\
\hline $\begin{array}{l}\text { Chin-Wen } \\
\text { Liao, Yu- } \\
\text { Cheng Liao, } \\
\text { Yao-Tsung } \\
\text { Chiang, Hui- } \\
\text { Fen } \quad \text { Wu, } \\
\text { Yun-Hua Liao } \\
-2016\end{array}$ & $\begin{array}{l}\text { Construir in- } \\
\text { dicadores de } \\
\text { competência, inte- } \\
\text { grando a aprendi- } \\
\text { zagem baseada em } \\
\text { investigação no es- } \\
\text { tudo de projeto dos } \\
\text { alunos em escolas } \\
\text { de ensino médio } \\
\text { profissionalizante }\end{array}$ & AHP, Delphi & $\begin{array}{l}\text { Construção } \text { de } 20 \\
\text { indicadores de com- } \\
\text { petência e } 79 \text { itens de } \\
\text { competência }\end{array}$ & $\begin{array}{l}\text { O estudo descobriu que o } \\
\text { instrutor e os alunos que } \\
\text { se concentram no pro- } \\
\text { cesso de aprendizagem } \\
\text { por investigação não são } \\
\text { totalmente iguais. Pes- } \\
\text { quisas relativas também } \\
\text { apontam os fatores que } \\
\text { podem afetar a imple- } \\
\text { mentação da aprendiza- } \\
\text { gem baseada em inves- } \\
\text { tigação }\end{array}$ \\
\hline $\begin{array}{l}\text { Tanty Oktavia, } \\
\text { Evaristus Didik } \\
\text { Madyatmadja - } \\
2018\end{array}$ & $\begin{array}{l}\text { Propor um modelo } \\
\text { para facilitar a ins- } \\
\text { tituição de ensino } \\
\text { superior na deter- } \\
\text { minação da área } \\
\text { de concentração de } \\
\text { alunos e testar sua } \\
\text { consistência }\end{array}$ & $\begin{array}{l}\text { AHP, TOP- } \\
\text { SIS (Order } \\
\text { Preference by } \\
\text { Similarity to } \\
\text { Ideal Solution) }\end{array}$ & $\begin{array}{l}\text { Baseado nos testes de } \\
\text { precisão que foram fei- } \\
\text { tos, o modelo apresen- } \\
\text { tou uma taxa de pre- } \\
\text { cisão de } 67 \%\end{array}$ & $\begin{array}{l}\text { O sistema de suporte à } \\
\text { decisão precisa ser avali- } \\
\text { ado de maneira mais de- } \\
\text { talhada e também requer } \\
\text { mais amostras }\end{array}$ \\
\hline $\begin{array}{l}\text { Ahsan M, } \\
\text { Setiyaningsih } \\
\text { W, Susilowati } \\
\text { M, Dijaya R, } \\
\text { Tjahjanti PH - } \\
2019\end{array}$ & $\begin{array}{l}\text { Decidir e classificar } \\
\text { a inteligência do } \\
\text { aluno com base } \\
\text { em inteligência } \\
\text { múltipla }\end{array}$ & $\begin{array}{l}\text { AHP, TOPSIS, } \\
\text { WP (Weighted } \\
\text { Product), SAW } \\
\text { (Simple Addi- } \\
\text { tive Weighting } \\
\text { Method), } \\
\text { ELECTRE } \\
\text { (ELimina- } \\
\text { tion Et Choix } \\
\text { TRADITIO- } \\
\text { NAL REAL) }\end{array}$ & $\begin{array}{l}\text { Criação de um método } \\
\text { de decisão que pode } \\
\text { representar as decisões } \\
\text { de um especialista } \\
\text { na determinação da } \\
\text { inteligência infantil e } \\
\text { também podem ajudar } \\
\text { um professor orien- } \\
\text { tador a ver como os } \\
\text { alunos aprendem }\end{array}$ & $\begin{array}{l}\text { Os instrumentos utiliza- } \\
\text { dos no estudo precisam } \\
\text { ser revistos para obter os } \\
\text { resultados que condizem } \\
\text { com a realidade }\end{array}$ \\
\hline
\end{tabular}




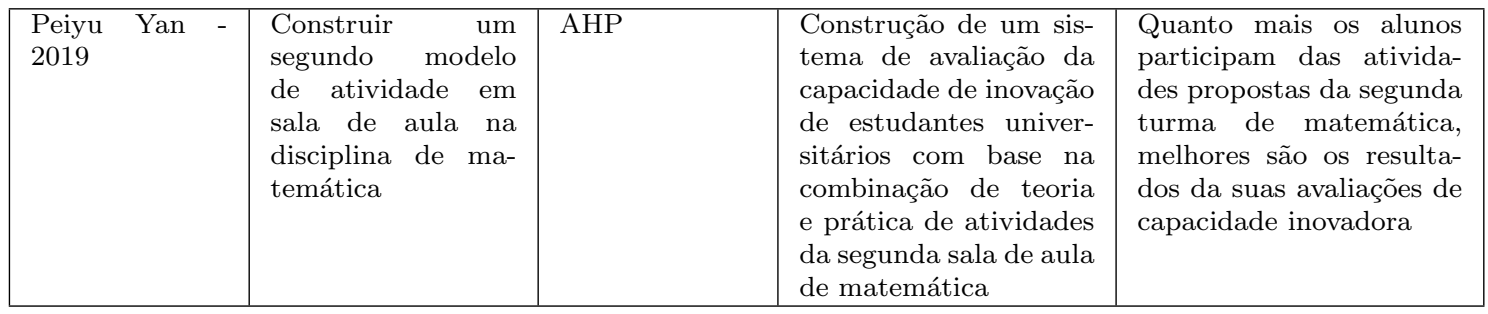

os trabalhos também apresentaram outros métodos (conjuntos, auxiliares ou comparativos) que podem ser conferidos através da Figura 1, que apresenta a quantidade de vezes em que cada um desses métodos foi encontrado.

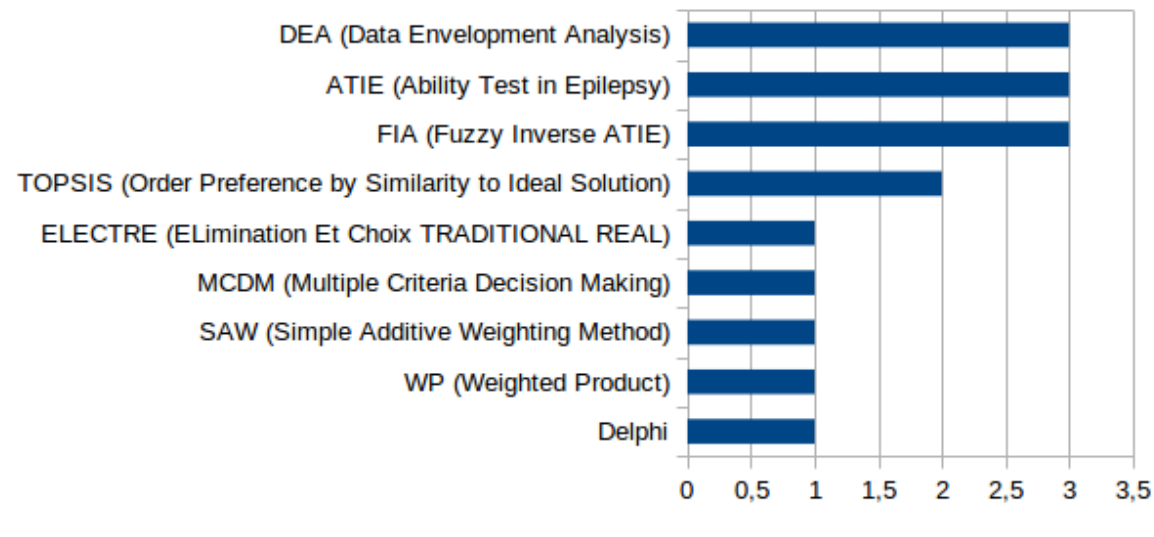

número de ocorrências

Figura 1: Outros métodos adotados nos trabalhos

\section{Discussão e conclusões}

A partir dos resultados apresentados na revisão de literatura podemos perceber que há um vasto campo de pesquisa envolvendo a teoria das Inteligências Múltiplas e Processo de Análise Hierárquica. Para o uso do método de AHP na teoria das MI foram encontrados trabalhos para as áreas de Saúde e Educação. Porém, algumas propostas de aplicações do método de AHP em conjunto com a teoria das MI poderiam ser empregadas em outras áreas do conhecimento como: Linguística, Letras, Artes, Engenharias, Ciências Exatas e da Terra, Humanas, Biológicas, Agrárias e Sociais Aplicadas.

Como podemos verificar, apenas sete trabalhos empregaram o Processo de Análise Hierárquica (AHP) (Analytic Hierarchy Process) na teoria das Inteligências Múltiplas (MI) (theory of Multiple Intelligences) ou, também, utilizaram a teoria das MI como base para qualquer tipo de problema que envolvesse o método AHP.

\section{Referências}

[1] Ahsan, M., Setiyaningsih, W., Susilowati, M., Dijaya, R. and Tjahjanti, P.H. Selecting multiple intelligences on children with weighted product, analytical hierarchy process, simple additive weighting and TOPSIS, Journal of Physics: Conference Series, 077033, 2019. DOI: 10.1088/1742-6596/1402/7/077033. 
[2] Armstrong, T. Multiple intelligences in the classroom. Ascd, Virginia, 2009.

[3] Gardner, H. Frames of mind: the theory of multiple intelligences. Basic Books, New York, 1983.

[4] Gardner, H. Inteligências múltiplas: a teoria na prática. Artmed, Porto Alegre, 1995.

[5] Gardner, H., Chen, J.-Q. e Moran, S. Inteligências múltiplas ao redor do mundo. Artmed, Porto Alegre, 2010.

[6] Kitchenham, B. Procedures for performing systematic reviews, NICTA Technical Report, 33:1$26,2004$.

[7] Liao, C.-W., Liao, Y.-C., Chiang, Y.-T., Wu, H.-F. and Liao, Y.-H. Integrating scientific inquiry learning into project course of vocational high schools to construct and verify core competency indicators, International Journal of Information and Education Technology, 6:836-842, 2016. DOI: 10.7763/IJIET.2016.V6.802.

[8] Nolen, J. Multiple intelligences in the classroom, Education, 124:115-, 2003.

[9] Oktavia, T. and Madyatmadja, E.D. Decision Support System to Help in Determining the Study Program Concentration for Higher Education Institution, Journal of Telecommunication, Electronic and Computer Engineering (JTEC), 10:1-9, 2018.

[10] Rezaie, V, Ahmad, T, Maan, N, Awang, S.R. and Khanmohammadi, M. A conceptual framework for ranking the multiple intelligences of people with epilepsy, International Conference on Statistics in Science, Business and Engineering (ICSSBE), 2012. DOI: 10.1109/ICSSBE.2012.6396540.

[11] Rezaie, V., Ahmad, T., Maan, N., Awang, S.R. and Khanmohammadi, M. Ranking the multiple intelligences of people with epilepsy using analytical hierarchy process and data envelopment analysis, Journal of Emerging Technologies in Web Intelligence, 5:98-106, 2013. DOI: 10.4304/jetwi.5.2.98-106.

[12] Rezaie, V., Ahmad, T., Awang, S.R., Khanmohammadi, M. and Maan, N. Evaluation of the Performance of Multiple Intelligence for People with Epilepsy, MATEMATIKA: Malaysian Journal of Industrial and Applied Mathematics, 30:21-29, 2014. DOI: 10.11113/matematika.v30.n0.736.

[13] Saaty, T. L. What is the analytic hierarchy process?, Mathematical models for decision support, Springer, Berlin, Heidelberg, pages 109-121, 1988. DOI: 10.1007/978-3-642-83555-1_5

[14] Saaty, T.L. How to make a decision: the analytic hierarchy process, European Journal of Operational Research, 48:9-26, 1990. DOI: 10.1016/0377-2217(90)90057-I.

[15] Saaty, T.L. Decision making with the analytic hierarchy process, International Journal of Services Sciences, 1:83-98, 2008. DOI: 10.1504/IJSSci.2008.01759.

[16] Sandelowski, M., Docherty, S. and Emden, C. Qualitative metasynthesis: Issues and techniques, Research in Nursing \& Health, 20:365-371, 1997.

[17] Vaidya, O.S. and Kumar, S. Analytic hierarchy process: An overview of applications, European Journal of Operational Research, 169:1-29, 2006. DOI: 10.1016/j.ejor.2004.04.028.

[18] Visser, B. A., Ashton, M. C. and Vernon, P. A. Beyond g: Putting multiple intelligences theory to the test, Intelligence, 34:487-502, 2006. DOI: 10.1016/j.intell.2006.02.004.

[19] Yan, P. The Second Class of Applied Undergraduate Mathematics and the Practice of College Students' Innovative Ability Cultivation, 5th International Conference on Social Science and Higher Education (ICSSHE), 2019. DOI: 10.2991/icsshe-19.2019.242. 